\title{
Highly uniform arrays of epitaxial Ge quantum dots with interdot spacing of $50 \mathrm{~nm}$
}

\author{
Christopher J. Duska and Jerrold A. Floro ${ }^{\text {a) }}$ \\ Department of Materials Science and Engineering, University of Virginia, Charlottesville, VA 22904, USA
}

(Received 25 March 14; accepted 15 August 14)

\begin{abstract}
Periodic, highly uniform arrays of dome-like Ge quantum dots (QDs) with $50 \mathrm{~nm}$ interdot pitch have been achieved on $\mathrm{Si}$ (001). The Si surface was patterned using ultra-low-dose focused ion beam and defect-selective etching, resulting in a continuously height-modulated, "egg-carton" morphology. The directed self-assembly process is robust, occurring across a range of ion doses, growth temperatures, and deposition rates. By selectively etching off the Ge dots to reveal the underlying Si surface just prior to Ge growth, we showed that Ge QDs preferentially formed on crowns (regions of negative curvature) rather than pits (regions of positive curvature) as is mostly seen in the literature. The width of the QD size distribution mimics that of the underlying substrate pattern, indicative of a complete lack of coarsening during the Ge growth, despite the small length scales, and extensive mass transport leading to QD formation.
\end{abstract}

\section{INTRODUCTION}

The integration of coherently strained epitaxial quantum dots (QDs) into semiconductor devices requires size and position control for device functionality. Surface templating to direct self-assembly of the site-controlled QD arrays can provide precise positioning and improved size homogeneity versus randomly nucleated QDs. ${ }^{1}$ Additional benefits to patterning include the potential to increase the areal density of QDs, the use of the 2D ordered QD array as a "seed layer" to create 3D ordered QD arrays, ${ }^{2}$ and ultimately the exploitation of interdot electronic interactions to create materials with novel, precisely tailored properties. ${ }^{3,4}$ Various surface features have been used to direct QD nucleation including pits, ${ }^{5-7}$ stripes, ${ }^{5,8}$ mesas, ${ }^{9,10}$ and oxide windows, ${ }^{11}$ which are formed via techniques including lithography, nanoindentation, and ion-beam processing. The reported growth experiments cover a wide range of pattern densities, growth temperatures, and areal coverage, yielding highly uniform arrays of ordered QDs. For the $\mathrm{Ge} / \mathrm{Si}$ (001) heteroepitaxial system, pattern spacings (pitches) are typically in the range of $100-500 \mathrm{~nm}$, corresponding to areal densities of $4 \times 10^{8}$ to $1 \times 10^{10} \mathrm{~cm}^{-2}$. In this work, we routinely attain $50 \mathrm{~nm}$ interdot pitch (center-tocenter site spacing), resulting in well-ordered arrays of Ge QDs with reasonably narrow size distribution. Preliminary QD patterns at $35 \mathrm{~nm}$ pitch were attained, but with reduced homogeneity. The challenges to directed self-assembly, associated with both initial pattern

\footnotetext{
a) Address all correspondence to this author.

e-mail: floro@virginia.edu

DOI: $10.1557 /$ jmr.2014.239
}

uniformity and Ostwald ripening of QDs, become severe as the length scales are reduced toward $10 \mathrm{~nm}$, where confinement and exchange coupling between QDs could provide interesting and useful tailoring of the electronic structure. $^{12-17}$

Heteroepitaxial $\mathrm{Ge}$ islands form to partially relax the $4 \%$ lattice mismatch strain with the $\mathrm{Si}$ substrate. QD formation requires growth conditions where adatom diffusion lengths match or exceed the intrinsic length scale, $\lambda \approx \Delta \gamma / \mathrm{M}^{2}$, where $\Delta \gamma$ is the surface energy change when QDs form, and $\mathrm{M} \varepsilon^{2}$ is the elastic energy density in the Ge due to the lattice mismatch. ${ }^{18}$ At the growth temperatures used here, after the formation of a 3-4 monolayer (ML) thick planar wetting layer, pyramidal Ge QDs form on a planar substrate, followed by the so-called dome clusters. ${ }^{19}$ At significantly higher temperatures, domes may actually form first. ${ }^{20}$ Surface templating ostensibly creates a locally varying chemical potential through modification of the surface curvature (or step density), surface strain, and/or surface chemistry. ${ }^{21}$ A large body of work specifically examines the effects of the changing surface morphology on the preferred nucleation of Ge QDs. However, the actual siting of the dots, e.g., inside or outside of pits, on top or on bottom of ridges, is surprisingly variable. While some clarity is beginning to emerge, it is clear that much remains to be understood, especially when the pattern pitch approaches, or is smaller than, the QD intrinsic length scale, $\lambda$.

Here we employ a $\mathrm{Ga}^{+}$focused ion beam (FIB) and defect-selective wet chemical etching to create a continuously height-modulated (quasi-sinusoidal) surface, rather than the discrete pits or ridges used in the existing literature. This modulated surface has a profound effect 
on QD nucleation. FIBs have been used earlier to template $\mathrm{Ge} \mathrm{QDs}^{7}$ as well as other Ge nanostructures. ${ }^{22}$ They possess unique advantages compared to photolithographic techniques including the direct writing of patterns, i.e., resist or pattern transfer are not required, and the ability to rapidly write complex, asymmetric patterns without the need for a mask. High-fidelity pattern replication has been reported for Ge QDs grown on FIB-patterned surfaces, although loss of fidelity was reported below $100 \mathrm{~nm}$ pitch due in part to competition between adjacent sites. ${ }^{23}$

We use an ultra-low-dose FIB to pattern $\mathrm{Si}(001)$, and relatively low growth temperatures $\left(450-550^{\circ} \mathrm{C}\right)$, to obtain Ge QD arrays with high areal density, $4 \times 10^{10}$ dots $/ \mathrm{cm}^{2}$, and low site vacancy fractions. There is a correlation between QD growth fidelity and pattern dose, which is also sensitive to the growth temperature. Unlike previous reports, our QDs do not nucleate within the pits, but in the 4-fold regions between the pits, referred to here as "crowns". Due to the high density of the patterns, the interpit regions are not (001) terraces, but have nonzero curvature. These crowns act as preferred nucleation sites. Potential mechanisms are discussed in the context of existing results and theories.

\section{EXPERIMENT}

$\mathrm{Si}$ (001) wafers with $<0.1^{\circ}$ miscut were cleaned with a $4: 1 \mathrm{H}_{2} \mathrm{SO}_{4}: \mathrm{H}_{2} \mathrm{O}_{2}$ solution, stripped in $5 \% \mathrm{HF}$, and passivated in UV ozone prior to loading in a FEI Helios Nanolab $650 \mathrm{Ga}^{+}$FIB system. The wafers were oxygen plasma cleaned in situ prior to patterning. The FIB was operated with an accelerating voltage of $30 \mathrm{kV}$ and emission current of $1.1 \mathrm{pA}$ with a manufacture specified beam diameter of $7 \mathrm{~nm}$. To ensure a circularly focused beam, the beam was focused and destigmatized at a magnification of $150 \mathrm{kx}$. The beam circularity was checked by imaging a reference pattern in a scanning electron microscope (SEM) mode. All patterns for this work consist of FIB-illuminated sites on a square lattice. Within a $100 \mu \mathrm{m} \times 100 \mu \mathrm{m}$ field we wrote an array of patterns of various doses ranging from 700-69 K ions/ site and lattice spacings typically 50, 35, and $20 \mathrm{~nm}$. The individual patterns contain as many as $500 \mathrm{~K}$ sites.

The patterned wafers were cleaned again with a modified IMEC/Shiraki process ${ }^{24}$ and then stripped in a $5 \% \mathrm{HF}$ solution prior to loading into an ultra-high vacuum (UHV) molecular beam epitaxy $(\mathrm{MBE})$ chamber $\left(P_{\text {base }}=1 \times\right.$ $10^{-10}$ Torr). In the MBE chamber the sample was radiatively heated to $280{ }^{\circ} \mathrm{C}$ for $30 \mathrm{~min}$ to degas. The sample was further heated to $550{ }^{\circ} \mathrm{C}\left(33^{\circ} \mathrm{C} / \mathrm{min}\right)$ for $5 \mathrm{~min}$ to desorb $\mathrm{H}$. The surface structure was monitored with reflection high-energy electron diffraction throughout the process. The substrate temperature was then reduced to $450{ }^{\circ} \mathrm{C}\left(500,550\right.$, and $625{ }^{\circ} \mathrm{C}$ in subsequent growths). Ge of $5 \mathrm{ML}$ thickness was deposited via magnetron sputtering in 5 mTorr of getter- and LN2purified Ar at a rate of $0.11 \AA / \mathrm{s}$ directly on the pattern without any Si buffer layer.

To directly compare the resulting Ge QD formation and the underlying surface pattern, the QDs were first characterized by atomic force microscopy (AFM). For the sample grown at $450{ }^{\circ} \mathrm{C}$, the Ge was then selectively etched by submerging the wafer in $65^{\circ} \mathrm{C}$ deionized water for $2 \mathrm{~h}$, dried by dry $\mathrm{N}_{2}$, and then rescanned by AFM. The AFM was performed with a NT-MDT Solver Pro-M in semicontact mode using NSG01/NSG10 tips with a typical radius of $6 \mathrm{~nm}$. The sample patterns were intentionally misaligned relative to the AFM scan direction to reduce flattening artifacts between the QDs. Select specimens were also examined using SEM.

\section{RESULTS}

AFM images of the as-written FIB patterns reveals net sputtering, i.e., direct formation of a milled pit, only at doses of $69 \mathrm{~K}$ ions/site and above, as shown in Fig. 1(a), whereas at $34 \mathrm{~K}$ ions/site and below, raised surface features are present at the FIB-implanted sites, Fig. 1(b). This arises from the defect generation and $\mathrm{Ga}^{+}$implantation during patterning and subsequent oxidation of the affected regions. ${ }^{25}$ However, after wet chemical cleaning in preparation for MBE growth, the defective FIB sites are selectively etched, leading to the formation of

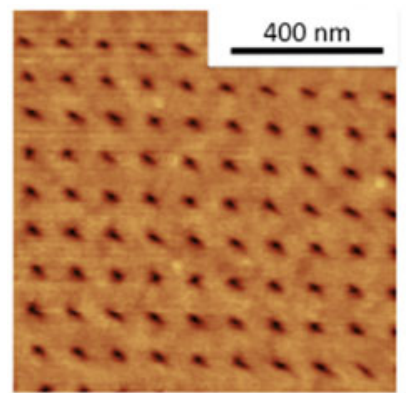

(a)

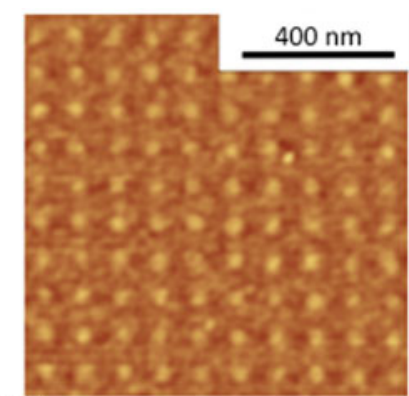

(b)

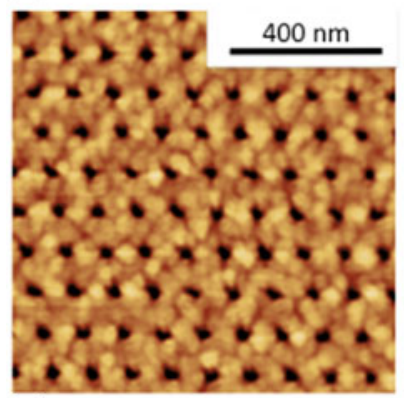

(c)

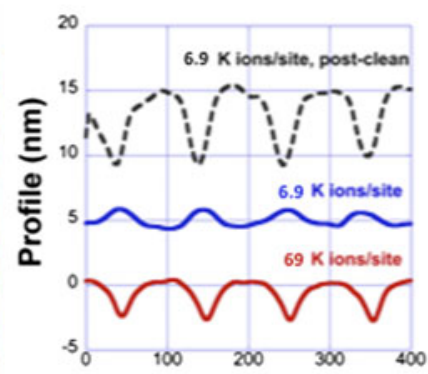

(d) Lateral Distance $(\mathrm{nm})$

FIG. 1. AFM micrographs of $\mathrm{Si}$ substrate surface after FIB exposure to (a) $69 \mathrm{~K}$ ions/site and (b) $34 \mathrm{~K}$ ions/site. (c) The surface of the sample in (b) after chemical etching. (d) Lateral line scans of the respective surface topographies $(\mathrm{nm})$. The scans are offset for clarity. 
nanoscale pits, see Fig. 1(c). The final depth and shape of the pit is in part determined by the number of oxidation/ stripping iterations.

The Ge MBE growth on the FIB patterns resulted in ordered QD arrays that reproduce the desired pattern to varying degrees. Figure 2 shows the AFM micrographs of the patterned Ge QDs with a $50 \mathrm{~nm}$ pitch. A select range of ion doses is shown wherein an optimal dose can be identified that produces the best apparent pattern fidelity. "Pattern fidelity" comprises three aspects: (i) location of the QD centroids with respect to the desired 2D lattice, (ii) the volume distribution of the QDs, which ideally would be a delta function, and (iii) site errors, including vacant sites and multiply populated sites. Each micrograph in Fig. 2 only covers a fraction of the patterned area, but the results are typical for the pattern as a whole. To provide a perspective, portions of a larger area scan are shown in Fig. 3; the extended ordering is evident. Long linear features are observed along the array axes 3(b), which are $<110\rangle$. These lines, where QDs are taller than their surroundings, have the topographical appearance of enhanced growth above misfit dislocations, which would be surprising however, given the small Ge thickness and height-modulated interface.

The distribution of QD volumes, sizes (both lateral and vertical), and mean positions of the QDs were determined from the AFM data using analysis techniques built into the software package, Gwyddion. ${ }^{26}$ The primary concern in determining the QD volume and size is the consistent choice of a threshold value representing the baseline height of the QDs. This analysis was performed on $1 \times 1 \mu \mathrm{m}$ AFM scans ( $\approx 400$ QDs), which were plane flattened and subjected to a band-pass filter to reduce high-frequency noise and low-frequency baseline variations. The threshold level was varied manually until just before the point when the QD base regions began to merge and percolate with their neighbors. The volumes and sizes are then calculated from this baseline. This will be an overestimate, especially of the volume, due to two effects: (i) tip convolution, which is significant on these length scales, and (ii) incorporation of some contribution from the substrate pattern itself. Regarding tip convolution, Fig. 4 compares the SEM and AFM micrographs from the same Ge QD pattern, where the lateral size of the QDs appears to be about half that obtained by AFM. Of course, the origin of contrast in secondary electron mode of the SEM will determine the apparent QD size; the actual QD diameter lies between the AFM and SEM values. Figure 5 shows the normalized QD volume distribution compared to the normalized crown volume. A key figure of merit included in the table is the scaled width of the distribution, defined here as $\sigma_{\mathrm{s}}=s /\langle V\rangle$, where $s$ is the standard deviation of the distribution, and $\langle V\rangle$ is the mean volume. Figure 6 plots $\langle V\rangle, \sigma_{\mathrm{s}}$, and $F_{\mathrm{m}}$ (the fraction of sites missing a QD) as a function of the ion dose. The doses of 3400, 2100, and 1400 ions/ site all yield similar results, with the best characteristics

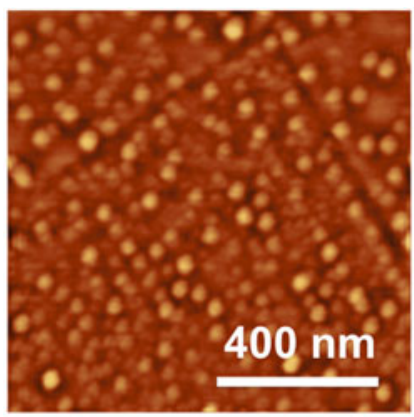

(a)

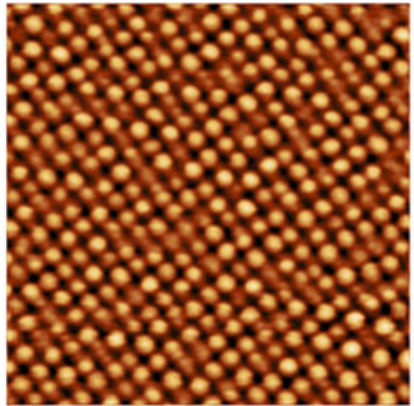

(d)

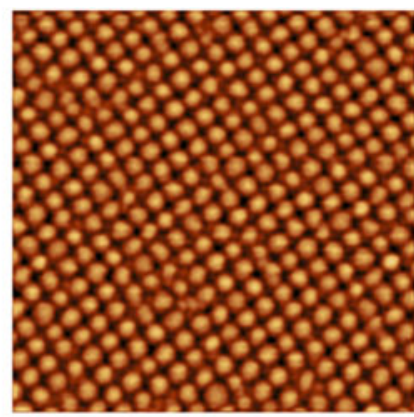

(b)

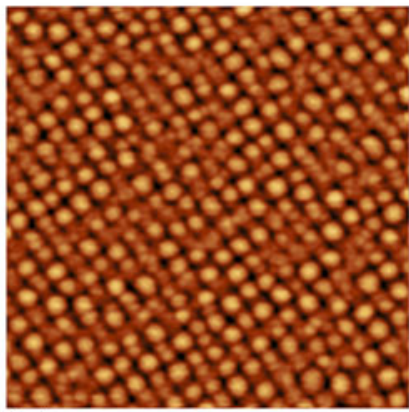

(e)

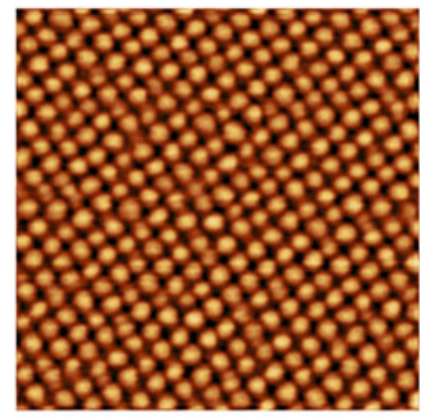

(c)

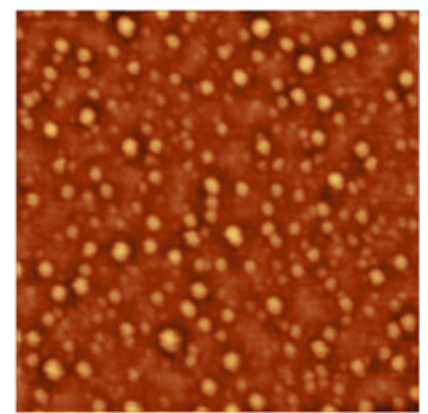

(f)

FIG. 2. $1 \times 1 \mu \mathrm{m}$ AFM micrographs showing the Ge QD morphology on patterns formed with $\mathrm{Ga}^{+}$doses of (a) 6900 ions/site, (b) 3400 ions/site, (c) 2100 ions/site, (d) 1400 ions/site, (e) 700 ions site, and (f) no pattern. All samples were from a single Ge growth at $450{ }^{\circ} \mathrm{C}$. 


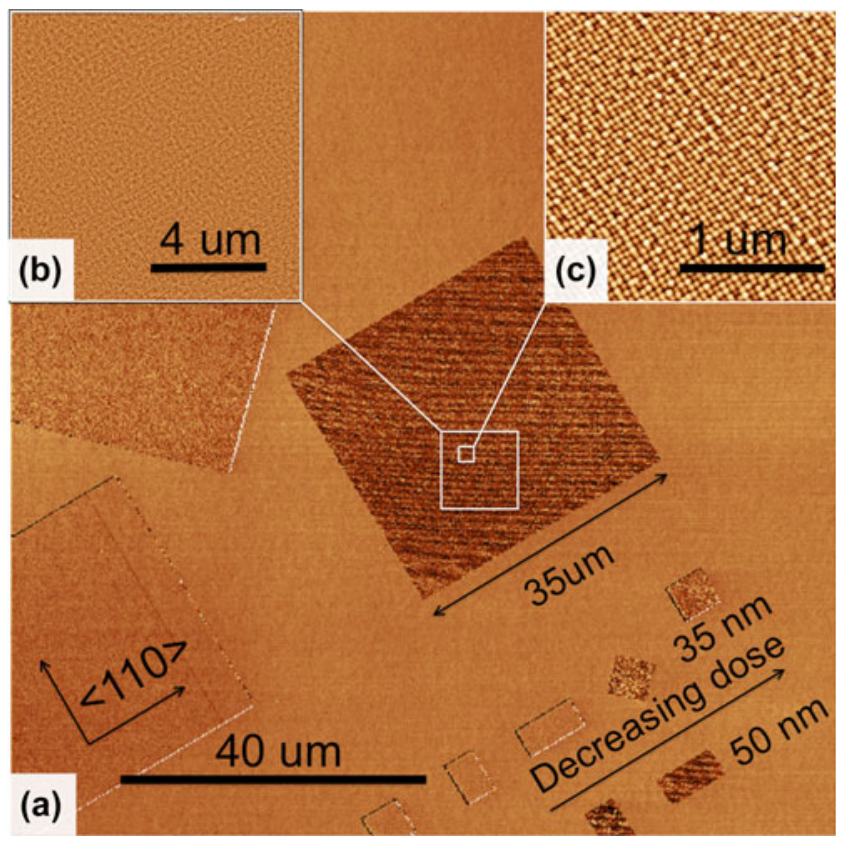

FIG. 3. (a) $100 \times 100 \mu \mathrm{m}$ AFM scan showing a typical array of patterns with varying pitch and dose. (b) $10 \times 10 \mu \mathrm{m}$ AFM scan from a $35 \times 35 \mu \mathrm{m}$ array of Ge QDs on a pattern formed using 700 ions/site; (c) shows a higher resolution, $2 \times 2 \mu \mathrm{m}$, scan from the same large area.

at 3400 ions/site, whereas at the 6900 ions/site, the QD pattern was so poor that statistical analysis was not attempted.

Pattern fidelity is also characterized by analyzing the 2D power spectral density function (PSDF), although this convolves both the positional accuracy and the size inhomogeneity. Peaks corresponding to the interdot distance are measured in reciprocal space and transformed to determine the nominal distance between the dots. The full width at half maximum (FWHM) of each is measured to get a sense of variation. The sources of error include miswriting of the array, random centering within each site, as well as the AFM drift. The mean interdot spacing is $52.4 \mathrm{~nm}$ with a standard deviation of $1.2 \mathrm{~nm}$ and an average FWHM of $4.7 \mathrm{~nm}$.

We examined the effect of the growth temperatures on the QD pattern characteristics in four separate growths. As shown in Fig. 7, an improvement in pattern fidelity is observed at $500{ }^{\circ} \mathrm{C}$. However, at 550 and $625{ }^{\circ} \mathrm{C}$, we observe the onset of coarsening and uniaxial coalescence of dots with clear broadening in volume distribution. Also, coarsening of the on-pattern arrays is clearly suppressed compared to those of the off-pattern QDs.

Figure 8 shows the morphology of the underlying $\mathrm{Si}$ surface after the Ge is selectively etched away. This allows direct correlation of the resultant QDs with the characteristics of the underlying substrate pattern that was present immediately prior to the growth. By examining the corner regions of each pattern (Fig. 9), we determine that QDs

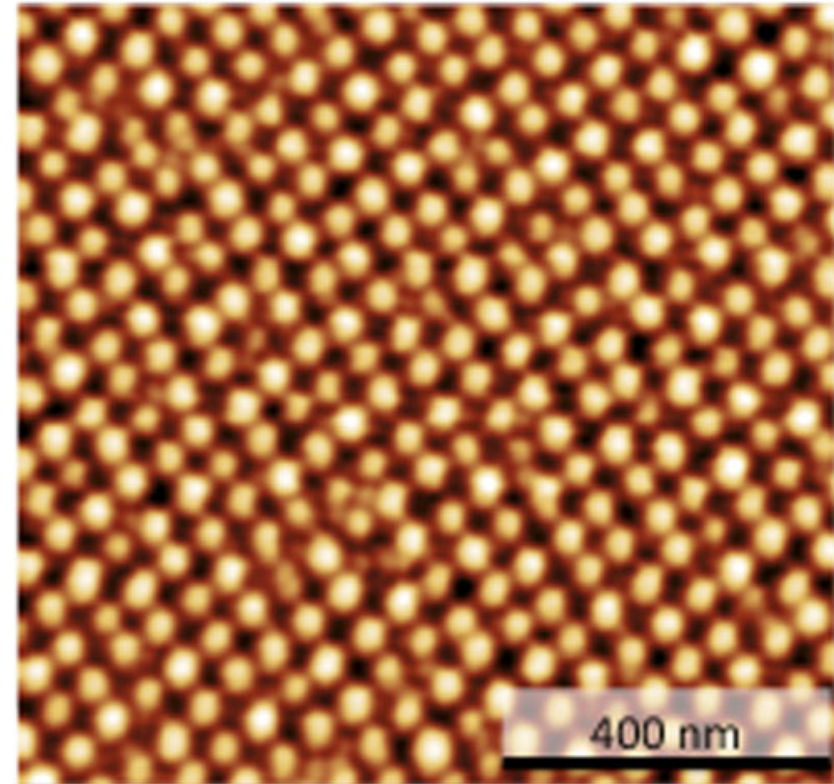

(a)

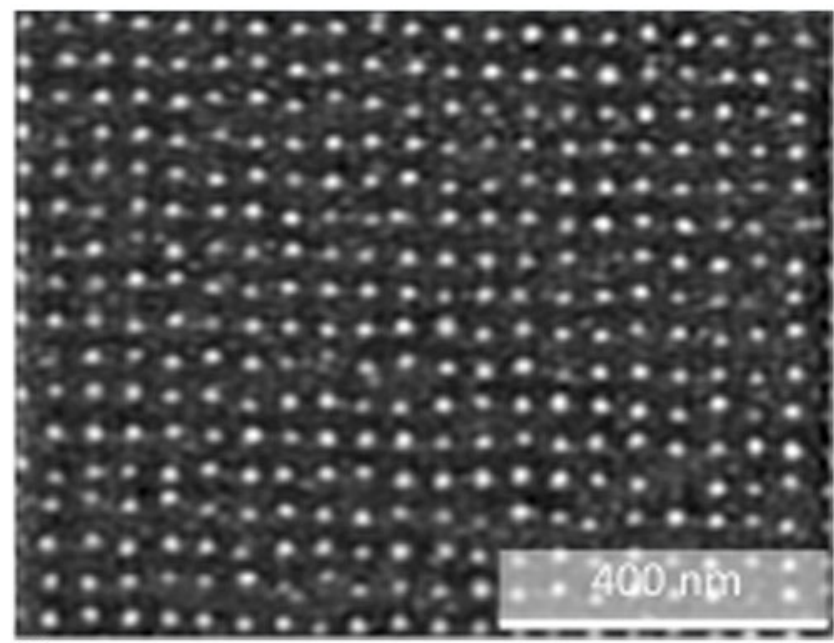

(b)

FIG. 4. Comparison of apparent QD lateral size in identical areas using (a) AFM and (b) SEM.

preferentially form in the interstitial locations of the pit lattice (a four-coordinated site), i.e., in the crown, or "ontop" positions of the pattern rather than on the on-bottom positions. This is in strong contrast to the previous reports on directed self-assembly of Ge using pit patterns where the formation occurs preferentially within the pits. ${ }^{23,27-30}$

Furthermore, we find that the patterns where optimal QD formation occurs are not in fact composed of isolated pits in the flat (001) terrace; rather, the surface appears to be continuously height modulated with no flat terrace regions between the pits. The striking resemblance between the before/after micrographs raises the question of whether Ge QDs are self-assembling, or instead, Ge just conformally coats the patterned Si surface. Figure 10 


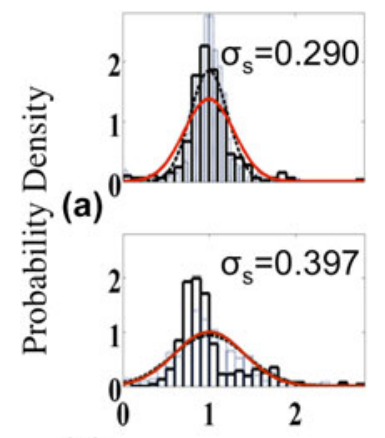

(c)

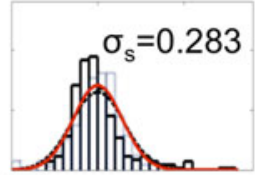

(b)

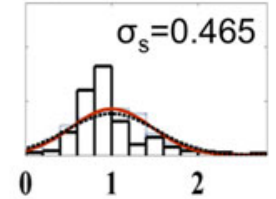

(d)

Volume $/<\mathrm{V}\rangle$

FIG. 5. Distributions of QD volumes, and the crown volumes, from the pattern after Ge was selectively removed. Data were obtained from $1 \times 1 \mu \mathrm{m}$ AFM scans on arrays patterned with (a) 3400 ions/site, (b) 2100 ions/site, (c) 1400 ions/site, and (d) 700 ions site. Both the histograms, a Gaussian fit for the QDs (red/solid), and crowns (black/dotted) are shown in each case.

(a)

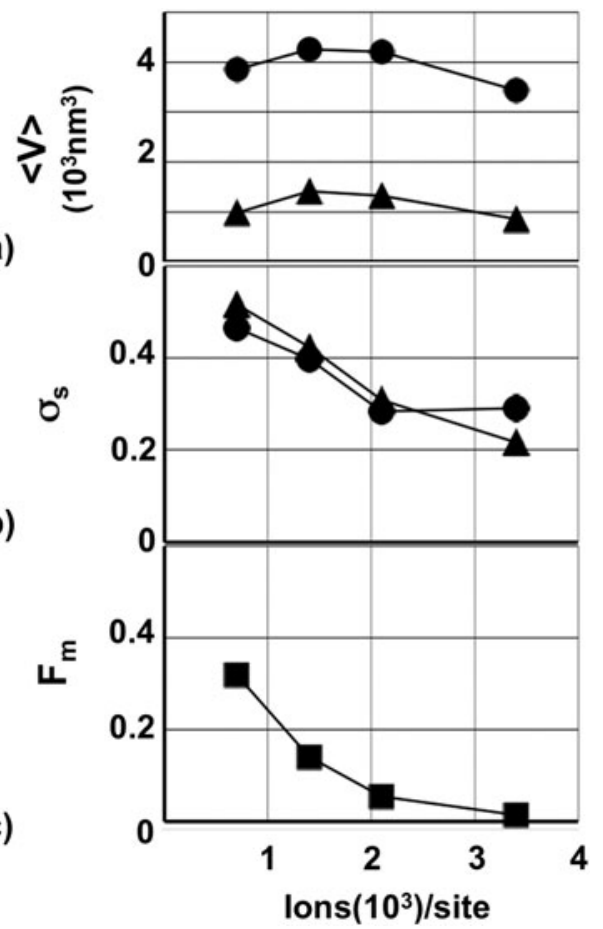

FIG. 6. Ion dose dependence of (a) mean feature volumes (QDs, circles, crowns, triangles) versus ion dose/site, (b) scaled distribution widths (QDs-circles and crowns-triangles) and (c) the QD site error fractions.

shows the AFM line scans from the micrographs in Fig. 8, overlaying typical scans from similar regions of the patterns. It is clearly seen that net $\mathrm{Ge}$ accumulation occurs to form QDs, with a large increase in height from $1-2 \mathrm{~nm}$ to $8-10 \mathrm{~nm}$. The local surface angle increases from maximum angles in the Si patterns of about $8^{\circ}$, to up to $30^{\circ}$ for the Ge QDs. For the lowest dose examined, 700 ions/site, AFM does reveal that isolated pits

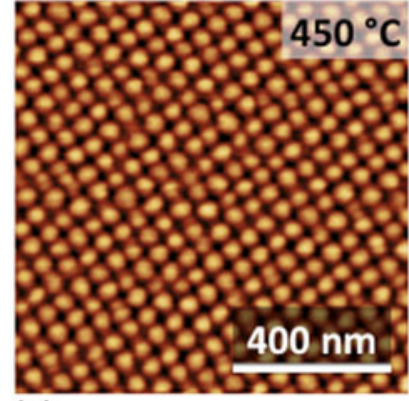

(a)

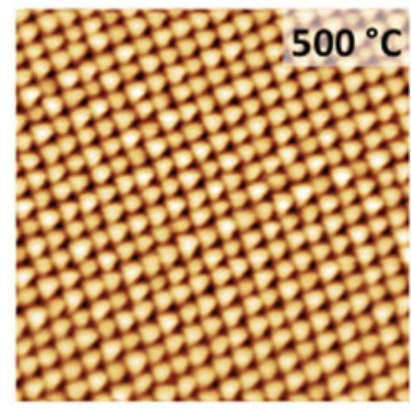

(c)

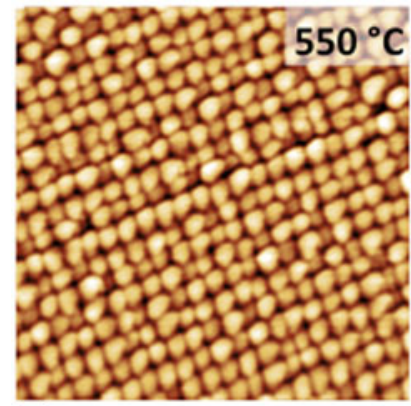

(e)

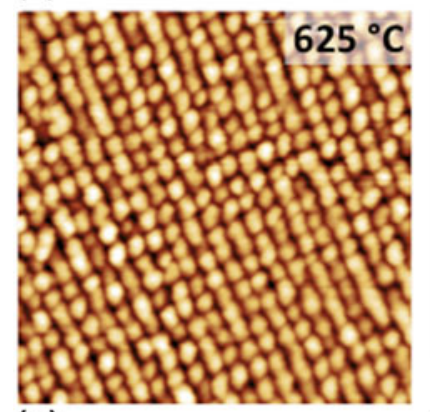

(g)

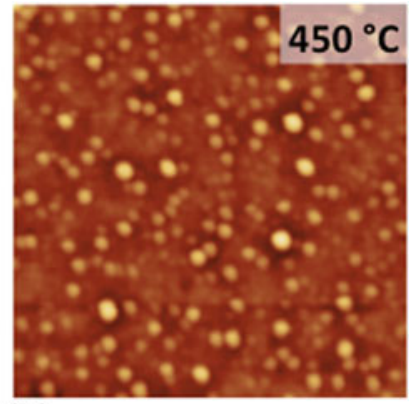

(b)

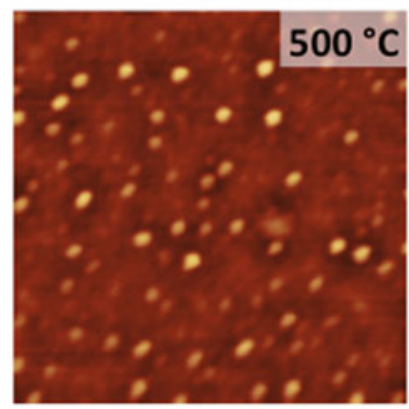

(d)

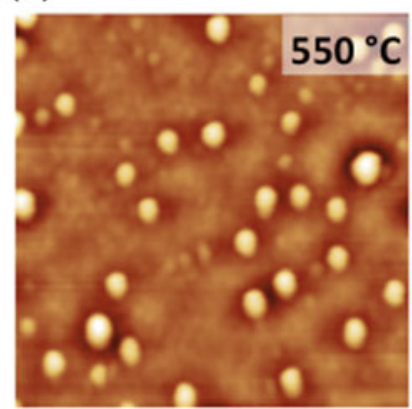

(f)

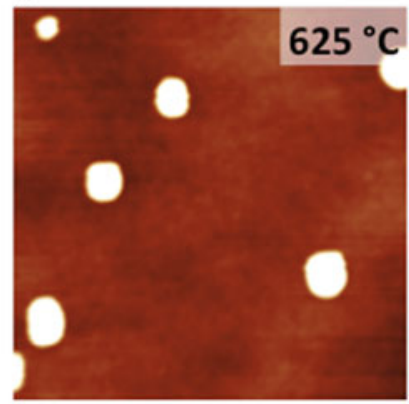

(h)

FIG. 7. $1 \times 1 \mu \mathrm{m}$ AFM micrographs of Ge QDs grown on patterns with 2100 ions/site dose (left column) and corresponding off-pattern QDs (right column) at (a)/(b) $450{ }^{\circ} \mathrm{C}$, (c) $/\left(\right.$ d) $500{ }^{\circ} \mathrm{C}$, (e)/(f) $550{ }^{\circ} \mathrm{C}$, and $(\mathrm{g}) /(\mathrm{h}) 625^{\circ} \mathrm{C}$.

separated by (001) terraces are formed, as shown in Fig. 10(d). However, the alignment and ordering of the QDs is actually rather poor in this pattern.

A quantitative analysis was also performed for the patterned Si surfaces after selective removal of the Ge. The scan data are from $1 \times 1 \mu \mathrm{m}$ regions in the center of each pattern. The surfaces were thresholded to measure 


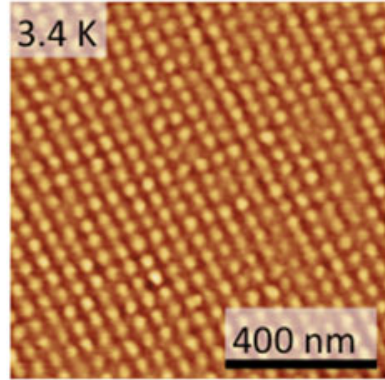

(a)

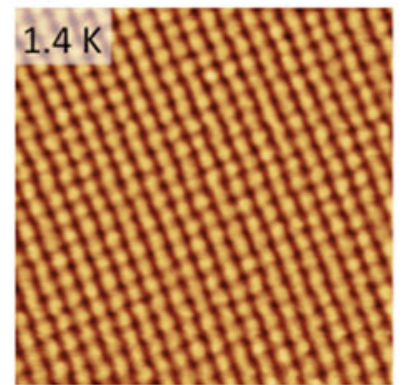

(c)

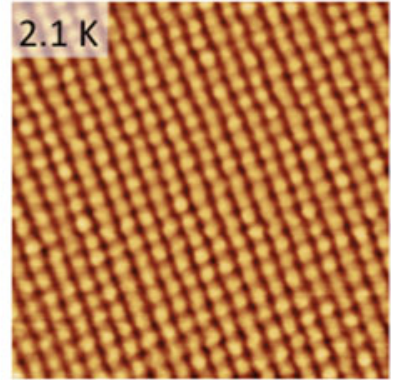

(b)

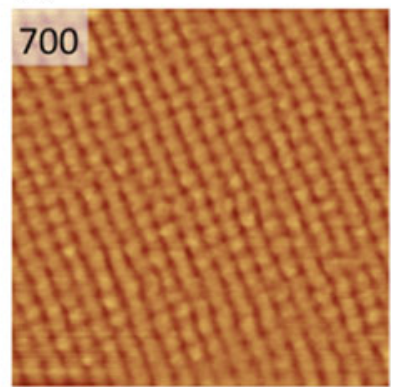

(d)

FIG. 8. AFM micrographs of the patterned Si surface upon which Ge QDs were grown at $450{ }^{\circ} \mathrm{C}$. Images were obtained by selectively etching off the Ge. (a) 3400 ions/site, (b) 2100 ions/site, (c) 1400 ions/site, and (d) 700 ions site.

the volumes of the crowns, as summarized in Fig. 6 . The crown periodicity was measured from the PSDF to have an overall average mean spacing of $50.00 \mathrm{~nm}$ and a standard deviation of $2.35 \mathrm{~nm}$. We examined the normalized volumetric distributions of both the crowns and their QD counterparts. The QD mean volumes track the crown volumes closely, suggesting that the diffusion between crown sites is highly constrained. While the normalized volumetric distribution width for the crowns monotonically narrows with increasing dose up to 3400 ions/site, the QD volumetric distribution width appears to saturate at $\sigma_{\mathrm{s}} \sim 0.30$. There is, however, a continued improvement in the pattern fidelity (i.e., decrease in site errors) along with the decrease in crown volume distribution width up to 3400 ions/site.

\section{DISCUSSION}

Many studies have shown that patterned arrays of isolated pits in the Si (001) surface effectively control the nucleation of Ge QDs. An overview can be found in Ref. 29 by Grydlik et al. and references therein. They show that the patterned pits can act as perfect sinks to form spatially ordered arrays of highly monodisperse Ge QDs (actually alloyed $\mathrm{GeSi}$ due to the intermixing during growth) as long as the Ge thickness is carefully limited, and the temperature and deposition rate are controlled so that adatom diffusion lengths match or exceed the pitch. This remarkable work has explored pitches from $300 \mathrm{~nm}$

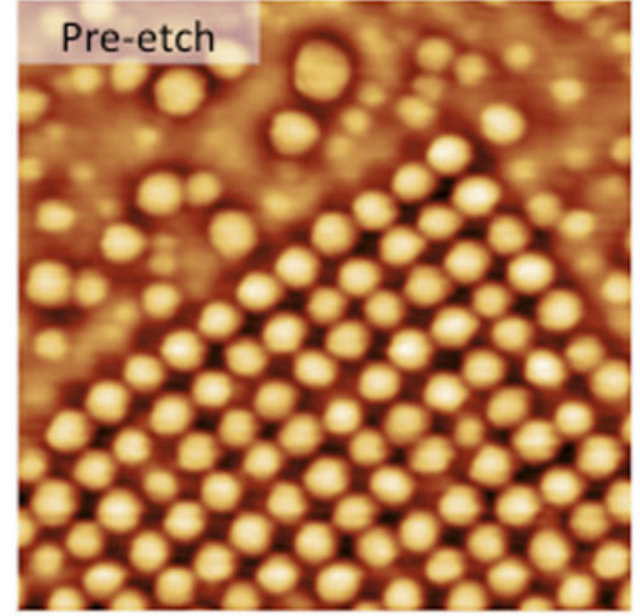

(a)

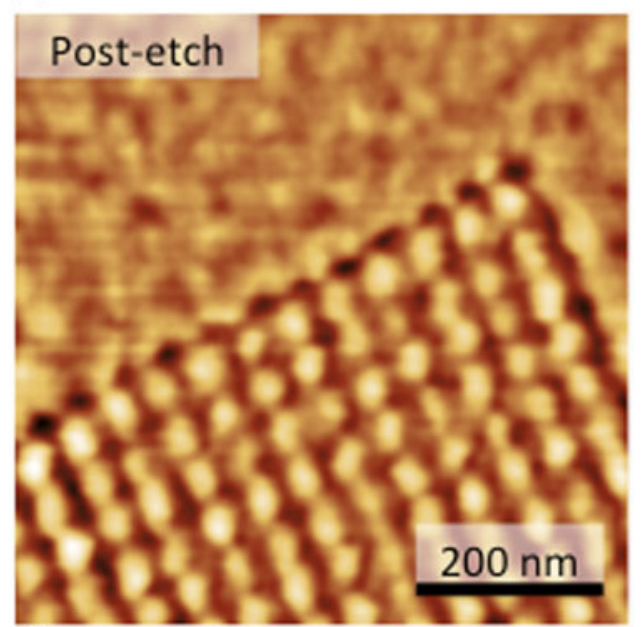

(b)

FIG. 9. $1 \times 1 \mu \mathrm{m}$ AFM micrographs of a corner of a pattern dosed at 3400 ions/site, (a) $5 \mathrm{ML}$ of Ge at $450{ }^{\circ} \mathrm{C}$, and (b) the same corner after Ge etch.

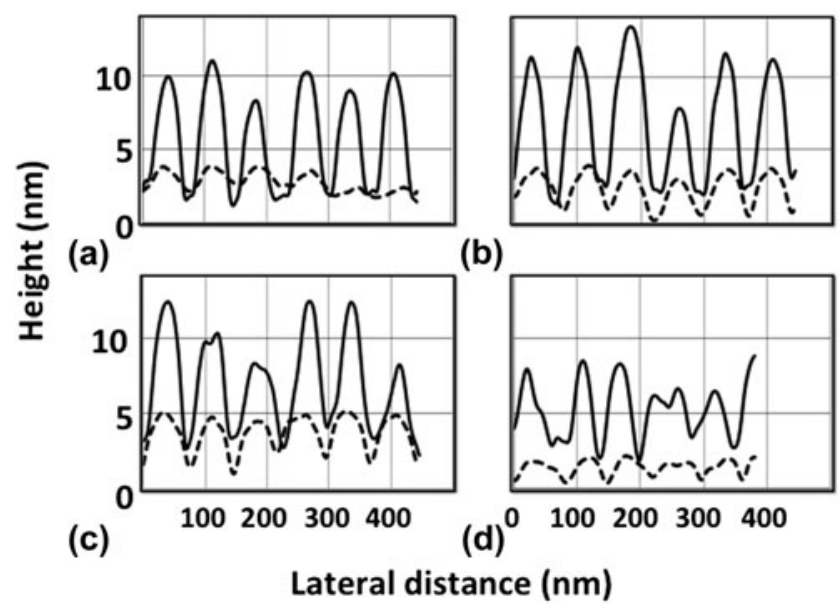

FIG. 10. AFM line scans comparing QDs (gray) to underlying substrate (dashed); all units are in $\mathrm{nm}$. (a) $3400 \mathrm{ions} / \mathrm{site}$, (b) 2100 ions/site, (c) 1400 ions/site, and (d) 700 ions site. 
to $3.4 \mu \mathrm{m}$ and has typically used growth temperatures well in excess of $600{ }^{\circ} \mathrm{C}$, and deposition rates of the order of $0.05 \AA / s$. These conditions provide diffusion lengths of order $10 \mu \mathrm{m}$, but also lead to extensive alloying of the QDs with the substrate. Grydlik et al. have identified at least 10 parameters that strongly affect the site-controlled QD growth during heteroepitaxy. Clearly, the feature sizes of the pattern, which can manifest as the pitch of a periodic pattern, the terrace size, pit size, the spectrum of surface angles etc., are critical parameters, especially in comparison to the intrinsic QD length scale and faceting.

Another key finding (see Vastola et al. $^{30}$ ) is that the pit sidewall angle has an optimal range of $5-18^{\circ}$ for symmetric QD formation at the pit bottom. Above about $32^{\circ}$, the QDs nucleated on the terraces at the edges of the pits (the pit angle here is actually the sidewall angle after Gewetting layer growth, which has a somewhat complex interplay with the size of the initial pit, the pit spacing, and the Ge coverage). Using finite element analysis of the elastic energy dependence on the pit angle, Vastola et al., achieved good agreement with the experimental results. This work demonstrates that under conditions where adatom mobility is not limiting, excessively shallow pits do not promote QD formation, intermediate angles lead to QD nucleation in the pit, while sufficiently steep pits lead to QD formation on the (001) terrace region immediately adjacent to the pit.

The pit-patterning approach has been extended to pitches at least as small as $90 \mathrm{~nm}$. The patterns were formed using extreme ultraviolet interference lithography, leading to pits only about $12 \mathrm{~nm}$ across and 3-4 nm deep. ${ }^{28}$ In this work, Ge was grown at $450{ }^{\circ} \mathrm{C}$ onto $50 \mathrm{~nm}$ $\mathrm{Si}$ buffers grown at $300{ }^{\circ} \mathrm{C}$. The resultant $\{105\}$-faceted pyramidal dots were about $45 \mathrm{~nm}$ in lateral size, i.e., the QDs were larger than the pits they nucleated upon ${ }^{2}$; highly monodisperse arrays still resulted, and these were subsequently used to create ordered three-dimensional arrays of QDs.

Portavoce et al. used the $\mathrm{Ga}^{+}$FIB patterning of $\mathrm{Si}$ in a UHV chamber attached to a transmission electron microscope (TEM). ${ }^{27}$ The growth of Ge QDs in the TEM was accomplished using chemical vapor deposition at $550{ }^{\circ} \mathrm{C}$. For optimal patterning dose and postannealing conditions, small Ge QDs $\approx 20 \mathrm{~nm}$ in diameter are obtained, on ultrasmall pits found (by ex situ AFM) to be only $10 \mathrm{~nm}$ in diameter, $1 \mathrm{~nm}$ deep, and $200 \mathrm{~nm}$ apart. This work also showed that nanoscale topography, rather than chemical effects from local Ga incorporation, directed the self-assembly.

Pascale et al. used ex situ $\mathrm{Ga}^{+}$FIB patterning coupled with subsequent MBE growth. ${ }^{31}$ The resulting surface after the buffer growth resulted in discrete pits surrounded by flat (001) terraces, although the size and shape of the pits are not further elucidated. For a $150 \mathrm{~nm}$ pitch, growth at $750{ }^{\circ} \mathrm{C}$ resulted in QDs forming in the 4-fold crowns (similar to what we observe); however, less than $25 \%$ of these sites were occupied. For growth at $550{ }^{\circ} \mathrm{C}$, QDs formed in the pits only, with a high occupation fraction. Based on the observed temperature dependence, and Monte Carlo modeling, they argued that the crown site has a lower total energy than the pit sidewall. However, their model does not actually consider QD formation in the pit bottom.

Using lithographic techniques, Chen et al. etched both pit and hill features into a $\mathrm{Si}(001)$ substrate. ${ }^{32}$ To a certain extent, the hill patterns resemble our patterned surfaces, albeit with larger pitches of $200-800 \mathrm{~nm}$, onto which $5 \mathrm{ML}$ of Ge was grown. Highly uniform, domeshaped islands were observed, located in the saddle points between adjacent hills, as opposed to the fourcoordinated minimum between adjacent hills (as seen here). However, the latter location is not actually a true pit, but an intersection between two orthogonal V-grooves. Nucleation on the crowns was not observed.

We have obtained highly dense, ordered arrays of Ge QDs on Si (001) surface-morphological patterns formed using low-dose FIB milling in conjunction with defect-selective etching. The resulting patterns, immediately prior to the $\mathrm{Ge}$ growth, are continuously height modulated, rather than being discrete pits separated by flat (001) terraces. Furthermore, our Ge QDs are larger in volume than the features that they grow upon. Several notable results will be discussed here: (i) there is an optimal range of ion dose where highly regimented patterns are observed; (ii) QD formation occurs on the four-coordinated "crowns" between pits, with strong site preference and near-unity occupation probability; (iii) Ostwald ripening appears to be largely suppressed despite the relatively small pitch of $50 \mathrm{~nm}$, leading to surprisingly homogeneous arrays where the width of the size distribution reflects that of the underlying pattern; and (iv) the results are similar for temperatures from 450 to $625{ }^{\circ} \mathrm{C}$.

Our results demonstrate that a range of ion doses per site can provide excellent patterning. Fidelity is lost for doses that are too large: presumably due to enlarged collision cascade volumes resulting in overlap and overetching of the highly defective volumes during wet chemical cleaning. This leads to a washout of the FIB pattern and loss of QD localization. At lower doses, the feature sizes are much smaller, and the distribution of feature sizes in the pregrowth template broadens significantly. This could arise from some coarsening of the pattern during the in situ heating prior to Ge growth, which would affect smaller feature sizes to a relatively larger degree than the larger features obtained at higher doses. In addition, lower doses might lead to varying degrees of defectivity and amorphization, resulting in more variability during wet chemical etching. 
The strong tendency for Ge QDs to form preferentially on the crowns versus the pits is a surprising result, given the robust literature indicating that pit nucleation is thermodynamically preferred across a wide range of growth and morphological conditions and for both lithographic and FIB-based patterning. The pits formed in this work have maximum sidewall angles within the range identified as ideal for QD siting within the pit. ${ }^{30}$ However, because of the small pitch and the degree of wet etching employed here, our surfaces are not flat (001) terraces with isolated pits, but rather are a continuously height-modulated, "egg-carton" surface. Two theoretical treatments have predicted that crown nucleation can be favorable under some circumstances.

Using a relatively simple 2D solution for the elastic energy associated with nucleation of QDs on a sawtoothpatterned substrate, Hu et al. show that the energy barrier to crown nucleation can become degenerate with pit nucleation. ${ }^{33}$ Although not emphasized explicitly by them, their model predicts that for each value of $\phi$, where $\phi$ is the facet angle of the sawtooth surface relative to the planar surface, there is a minimum surface energy anisotropy, $\alpha$, where the nucleation barrier disappears (the anisotropy in this model is essentially the difference between the maximum and minimum surface energies). This arises because the slope of the substrate surface forces the $\mathrm{Ge}$ wetting layer, initially assumed to be conformal, into a higher surface energy state. If the increase in surface energy is sufficiently large, then nucleation of the lower-energy facet on the QD becomes favorable, even if elastic relaxation is small (as it is on the crowns vis-à-vis the pits). Two issues arise in assessing the relevance of the $\mathrm{Hu}$ et al. model to our results. First, it predicts that crown and pit nucleations are, at best, equivalent. Since our results show a complete preference for crown nucleation, some other mechanism must break the symmetry between the nucleation sites. The second issue has to do with the nature of QD formation. Pit and crown nucleation only become equivalent under the condition where the nucleation barrier disappears, where it is not clear if the model remains relevant.

Preferred QD formation on crowns has been predicted by Aqua et al. using a detailed continuum model that specifically considers the roughening instability modes of strained films grown on 2D "egg-carton" patterns resembling those used here. ${ }^{34}$ Their model employs a nonlinear analysis that builds on their previous work in linear order. ${ }^{35,36}$ The model tracks the surface-diffusional flow in response to local chemical potential incorporating morphological strain relaxation and surface energy anisotropy, as well as the effects of wetting layer stabilization that can profoundly influence the 2D-3D transition. They plot a resultant phase space as a function of pattern periodicity and film thickness (valid once the film has reached a stationary morphological state at any given thickness).
A region of preferential crown formation occurs when (i) the substrate pattern wave length is $1-3 x$ that of the Asaro-Tiller-Grinfeld (ATG) instability wave length and (ii) when the film thickness is just above the critical wetting layer thickness where island formation first occurs. The ATG instability wave length is given by $\lambda_{\mathrm{ATG}}=$ $4 \pi \gamma_{\mathrm{w}} / 3 \mathrm{M} \varepsilon^{2}$ [Ref. 18], where $\gamma_{\mathrm{w}}$ is the Ge wetting layer surface energy and $M$ is the biaxial modulus of $\mathrm{Ge}$ strained to $\varepsilon=0.04$. Taking $\gamma_{\mathrm{w}}=61 \mathrm{meV} / \AA^{2}$ [Ref. 36] and $M=$ $152 \mathrm{GPa}^{38}$ gives $\lambda_{\mathrm{ATG}}=17 \mathrm{~nm}$. Hence our patterns are in the correct range of period, but somewhat thicker than the criteria established by $\mathrm{Xu}$ et al. for the preferential crown formation of QDs. This is nonetheless an intriguing result and bears further investigation.

The formation of dome-like Ge QDs with $25^{\circ}$ sidewall angles shows that significant mass transport occurs from the pits to the crowns. However, coarsening of the patterned Ge dots is minimal, as evidenced by the narrow size distribution, and more importantly, because the width of the QD volume distribution mimics that of the underlying Si pattern. This indicates that each crown captures flux only from the 2D unit cell defined by a square cell of four pits with a crown in the middle. The work reported in Grydlik et al. ${ }^{29}$ demonstrates that even when the Ge diffusion length far exceeds the 2D unit cell dimension, monodisperse, noncoarsened arrays are obtained. This is explained by a tendency of the pits to prevent outflux (thereby slowing coarsening). Furthermore, the deterministic nucleation of QDs on the patterns leads to an initial highly uniform distribution, creating a quasi-stationary state that does not coarsen due to the suppression of the local (or mean-field) driving force. In principle, this state could be destabilized by the presence of one island whose volume is significantly different from the rest of the distribution. We do have a nonzero areal density of QDs whose size is more than $3 \sigma$ outside the mean, but this does not seem to destabilize the array. It is possible that surface diffusion on the height-modulated $\mathrm{Si}$ surface produced here is retarded relative to the (001) terrace. This could arise, for example, from Erlich-Schwoebel (E-S) barriers. A small barrier of about $60 \mathrm{meV}$ has been suggested for unstrained Ge (001). ${ }^{39}$ However, at the growth temperatures used here, the effect of a small E-S barrier on dot formation is expected to be minimal in comparison with the strain. ${ }^{40}$ More work is needed to understand the apparent lack of coarsening.

\section{SUMMARY}

Periodic, highly uniform arrays of dome-like Ge QDs with $50 \mathrm{~nm}$ interdot pitch have been achieved on $\mathrm{Si}$ (001). The Si surface was patterned using ultra-low-dose FIB and defect-selective etching, resulting in a continuously height-modulated, "egg-carton" morphology. 
The directed self-assembly process is robust, occurring across a range of ion doses, growth temperatures, and with and without a Si buffer layer. By selectively etching off the Ge dots to reveal the underlying Si surface just prior to the Ge growth, we showed that Ge QDs preferentially formed on crowns (regions of negative curvature) rather than pits (regions of positive curvature) as is mostly seen in the literature. The width of the QD size distribution mimics that of the underlying substrate pattern, indicative of a complete lack of coarsening during the Ge growth, despite the small length scales, and clear mass transport leading to the QD formation. Much remains to be understood about the formation mechanism and growth kinetics; nonetheless, the robust process provides optimism that lateral length scales can be reduced even further, to where proximal interaction effects between the QDs can provide novel electronic or thermal transport behavior.

\section{ACKNOWLEDGMENTS}

We acknowledge Gopal Ramalingam, Ehsan Monazami Alipour, and Prof. Petra Reinke for their help with key low rate Ge QD growths, and Jean-Noël Aqua for discussions concerning his analysis of growth instabilities on patterned substrates. Funding was generously provided by the II-VI Foundation. Research was performed in part at the Center for Nanoscale Science and Technology (CNST) at the National Institute for Science and Technology (NIST), Gaithersburg, MD, with special thanks to Dr. Joshua Schumacher for his assistance with FIB patterning.

\section{REFERENCES}

1. Z. Zhong and G. Bauer: Site-controlled and size-homogeneous Ge islands on prepatterned $\mathrm{Si}$ (001) substrates. Appl. Phys. Lett. 84, 1922 (2004).

2. D. Grützmacher, T. Fromherz, C. Dais, J. Stangl, E. Müller, Y. Ekinci, H.H. Solak, H. Sigg, R.T. Lechner, E. Wintersberger, S. Birner, V. Holy', and G. Bauer: Three-dimensional Si/Ge quantum dot crystals. Nano Lett. 7, 3150-3156 (2007).

3. C.E. Pryor, M.E. Flatté, and J. Levy: Electrical manipulation of an electronic two-state system in Ge quantum dots. Appl. Phys. Lett. 95, 232103 (2009).

4. T. Fromherz, J. Stangl, R.T. Lechner, E. Wintersberger, G Bauer, V. Holy, C. Dais, E. Müller, H. Sigg, H.H. Solak, and D. Grützmacher: 3D SiGe quantum dot crystals: Structural characterization and electronic coupling. Int. J. Mod. Phys. B 23, 2836-2841 (2009)

5. Z. Zhong, A. Halilovic, and M. Mühlberger, F. Schäffler, and G. Bauer: $\mathrm{Ge}$ island formation on stripe-patterned $\mathrm{Si}(001)$ substrates. Appl. Phys. Lett. 82, 445 (2003).

6. F.M. Ross, M. Kammler, M.C. Reuter, and R. Hull: In-situ observations of self-assembled island nucleation on patterned substrates. Philos. Mag. 84, 2687-2702 (2004).

7. A. Karmous, A. Cuenat, A. Ronda, I. Berbezier, S. Atha, and R. Hull: Ge dot organization on Si substrates patterned by focused ion beam. Appl. Phys. Lett. 85, 6401 (2004).
8. T.I. Kamins and R.S. Williams: Lithographic positioning of selfassembled Ge islands on Si(001). Appl. Phys. Lett. 71, 1201 (1997).

9. T. Kitajima, B. Liu, and S.R. Leone: Two-dimensional periodic alignment of self-assembled $\mathrm{Ge}$ islands on patterned $\mathrm{Si}(001)$ surfaces. Appl. Phys. Lett. 80, 497 (2002).

10. G. Jin, J.L. Liu, S.G. Thomas, Y.H. Luo, K.L. Wang, and B-Y. Nguyen: Perfect alignment of self-organized Ge islands on pre-grown $\mathrm{Si}$ stripe mesas. Appl. Phys. A: Mater. Sci. Process. 70, 551-554 (2000).

11. T.S. Yoon, H-M. Kim, K-B. Kim, D.Y. Ryu, T.P. Russell, Z. Zhao, J. Liu, and Y-H. Xie: Study of growth behaviour and microstructure of epitaxially grown self-assembled Ge quantum dots on nanometer-scale patterned $\mathrm{SiO} 2 / \mathrm{Si}(001)$ substrates. Phys. Status Solidi 246, 721-724 (2009).

12. G. Katsaros, P. Spathis, M. Stoffel, F. Fournel, M. Mongillo, V. Bouchiat, F. Lefloch, A. Rastelli, O.G. Schmidt, and S. DeFrancheschi: Hybrid superconductor-semiconductor devices made from self-assembled $\mathrm{SiGe}$ nanocrystals on silicon. Nat. Nanotechnol. 5, 458-464 (2010).

13. K.L. Wang, D. Cha, J. Liu, and C. Chen: Ge/Si self-assembled quantum dots and their optoelectronic device applications. Proc. IEEE 95, 1866-1883 (2007).

14. K. Brunner: Si/Ge nanostructures. Rep. Prog. Phys. 65, 27-72 (2002).

15. K. Eberl, O.G. Schmidt, R. Duschl, O. Kienzle, E. Ernst, and Y. Rau: Self-assembling SiGe and $\mathrm{SiGeC}$ nanostructures for light emitters and tunneling diodes. Thin Solid Films 369, 33-38 (2000).

16. O.G. Schmidt, U. Denker, M. Dashiell, N.Y. Jin-Phillipp, K. Eberl, R. Schreiner, H. Grabeldinger, H. Schweizer, S. Christiansen, and F. Ernst: Laterally aligned $\mathrm{Ge} / \mathrm{Si}$ islands: A new concept for faster field-effect transistors. Mater. Sci. Eng., B 89, 101-105 (2002).

17. G. Fiedler and P. Kratzer: Theoretical prediction of improved figure-of-merit in $\mathrm{Si} / \mathrm{Ge}$ quantum dot superlattices. New J. Phys. 23, 2836-2841 (2013).

18. D. Srolovitz: On the stability of surfaces of stressed solids. Acta Metall. 37, 621-625 (1989).

19. G. Medeiros-Ribeiro, A. Bratkovski, T.I. Kamins, D. Ohlberg, and R.S. Williams: Shape transition of germanium nanocrystals on a silicon (001) surface from pyramids to domes. Science 279, 353-356 (1998)

20. M. Brehm, F. Montalenti, M. Grydlik, G. Vastola, H. Lichtenberger, N. Hrauda, M.J. Beck, T. Fromherz, F. Schäffler, L. Miglio, and G. Bauer: Key role of the wetting layer in revealing the hidden path of $\mathrm{Ge} / \mathrm{Si}(001)$ Stranski-Krastanow growth onset. Phys. Rev. B 80, 205321 (2009).

21. B. Yang, F. Liu, and M. Lagally: Local strain-mediated chemical potential control of quantum dot self-organization in heteroepitaxy. Phys. Rev. Lett. 92, 025502 (2004).

22. R. Hull, J. Floro, J. Graham, J. Gray, M. Gherasimova, A. Portavoce, and F.M. Ross: Synthesis and functionalization of epitaxial quantum dot nanostructures for nanoelectronic architectures. Mater. Sci. Semicond. Process. 11, 160-168 (2008).

23. M. Gherasimova, R. Hull, M.C. Reuter, and F.M. Ross: Pattern level assembly of Ge quantum dots on $\mathrm{Si}$ with focused ion beam templating. Appl. Phys. Lett. 93, 023106 (2008).

24. J. Kassim, C. Nolph, M. Jamet, P. Reinke, and J. Floro: $\mathrm{Ge}_{1-\mathrm{x}} \mathrm{Mn}_{\mathrm{x}}$ heteroepitaxial quantum dots: Growth, morphology, and magnetism. J. Appl. Phys. 113, 073910 (2013).

25. J. Graham, C.D. Kell, J.A. Floro, and R. Hull: Coupled effects of ion beam chemistry and morphology on directed self-assembly of epitaxial semiconductor nanostructures. Nanotechnology 22, 075301 (2011).

26. D. Necas and P. Klapetek: Gwyddion: An open-source software for SPM data analysis. Cent. Eur. J. Phys. 10, 181-188 (2012). 
27. A. Portavoce, M. Kammler, R. Hull, M.C. Reuter, and F.M. Ross: Mechanism of the nanoscale localization of Ge quantum dot nucleation on focused ion beam templated $\mathrm{Si}(001)$ surfaces. Nanotechnology 17, 4451-4455 (2006).

28. D. Grützmacher, C. Dais, L. Zhang, E. Müller, and H.H. Solak: Templated self-organization of SiGe quantum structures for nanoelectronics. Mater. Sci. Eng., C 27, 947-953 (2007).

29. M. Grydlik, G. Langer, T. Fromherz, F. Schäffler, and M. Brehm: Recipes for the fabrication of strictly ordered $\mathrm{Ge}$ islands on pit-patterned $\mathrm{Si}(001)$ substrates. Nanotechnology $\mathbf{2 4}$, 105601 (2013).

30. G. Vastola, M. Grydlik, M. Brehm, T. Fromherz, G. Bauer, F. Boioli, L. Miglio, and F. Montalenti: How pit facet inclination drives heteroepitaxial island positioning on patterned substrates. Phys. Rev. B 84, 155415 (2011).

31. A. Pascale, I. Berbezier, A. Ronda, and P.C. Kelires: Selfassembly and ordering mechanisms of Ge islands on prepatterned Si(001). Phys. Rev. B 77, 075311 (2008).

32. G. Chen, G. Vastola, H. Lichtenberger, D. Pachinger, G. Bauer, W. Jantsch, F. Schäffler, and L. Miglio: Ordering of Ge islands on hill-patterned Si (001) templates. Appl. Phys. Lett. 92, 113106 (2008).
33. H. Hu, H.J. Gao, and F. Liu: Theory of directed nucleation of strained islands on patterned substrates. Phys. Rev. Lett. 216102, 2-5 (2008).

34. J.-N. Aqua and X. Xu: Self-organization vs. directed growth of quantum dots. Phys. Rev. B (2014, accepted).

35. X. J.-N. Aqua, J. Aqua, and T. Frisch: Growth of a strained epitaxial film on a patterned substrate. Phys. Rev. B 14, (2014, submitted).

36. X. Xu, J. Aqua, and T. Frisch: Growth kinetics in a strained crystal film on a wavy patterned substrate. J. Phys. Condens. Matter 24, 045002 (2012).

37. O. Shklyaev, M. Beck, M. Asta, M. Miksis, and P. Voorhees: Role of strain-dependent surface energies in $\mathrm{Ge} / \mathrm{Si}(100)$ island formation. Phys. Rev. Lett. 94, 176102 (2005).

38. J.A. Floro, E. Chason, S.R. Lee, and G.A. Petersen: Biaxial moduli of coherent $\mathrm{Si}_{1-\mathrm{x}} \mathrm{Ge}_{\mathrm{x}}$ films on $\mathrm{Si}$ (001). Appl. Phys. Lett. 71, 1694 (1997).

39. B. Shin and M. Aziz: Modeling RHEED intensity oscillations in multilayer epitaxy: Determination of the Ehrlich-Schwoebel barrier in Ge(001) homoepitaxy. Phys. Rev. B 76, 165408 (2007).

40. Z-J. Liu and Y.G. Shen: Roughening kinetics of thin films in the presence of both stress and Ehrlich-Schwobel barrier. Appl. Phys. Lett. 83, 5404 (2003). 